07 DEPRESSION AND SEXUAL BEHAVIOUR AMONG MEN WHO HAVE SEX WITH MEN IN THE UK

${ }^{1}$ Ada Miltz* ${ }^{1}$ Alison Rodger, ${ }^{1}$ Janey Sewell, ${ }^{1}$ Andrew Speakman, ${ }^{1}$ Andrew Phillips, ${ }^{1}$ Lorraine Sherr, ${ }^{1,2}$ Richard Gilson, ${ }^{3}$ David Asboe, ${ }^{4}$ Nneka Nwokolo, ${ }^{5}$ Christopher Scott, ${ }^{3}$ Sara Day, ${ }^{6}$ Martin Fisher, ${ }^{6}$ Amanda Clarke, ${ }^{7}$ Jane Anderson, ${ }^{8}$ Rebecca O'Connell, ${ }^{9}$ Monica Lascar, ${ }^{10}$ Vanessa Apea, ${ }^{11}$ Rageshri Dhairyawan, ${ }^{12}$ Mark Gompels. 'University College London, London, UK; ${ }^{2}$ The Mortimer Market Centre, London, UK; ${ }^{3}$ John Hunter Clinic, London, UK; ${ }^{4}$ Dean Street Sexual Health Clinic, London, UK; ${ }^{5}$ West London Centre for Sexual Health, London, UK; ${ }^{6}$ Royal Sussex County Hospital, Brighton, UK; ${ }^{7}$ Homerton Hospital, London, UK; ${ }^{8}$ Newham Hospital, London, UK; ${ }^{9}$ Whipps Cross Hospital, London, UK; ${ }^{10}$ Barts Sexual Health Centre/The London Hospital, London, UK; ${ }^{11}$ Barking Hospital, London, UK; ${ }^{12}$ Southmead Hospital, Bristol, UK

10.1136/sextrans-2015-052126.7

Background/introduction In the UK, HIV transmission remains ongoing among men who have sex with men (MSM). Data on mental health and sexual behaviour is limited among MSM whose HIV-status is negative/unknown.

Aim(s)/objectives To describe the association of depressive symptoms with measures of condomless sex (CLS).

Methods AURAH (Attitudes to, and Understanding of, Risk of Acquisition of HIV) is a cross-sectional questionnaire study in 20 UK STI clinics. We included MSM recruited from May 2013January 2014 who reported anal sex in the past three months. Depressive symptoms were defined as a PHQ-9 score $\geq 10$. We examined the association of depressive symptoms with: CLS in the past three months with (i) $\geq 2$ partners (ii) discordant status partner(s) (unknown/HIV-positive) and self-reported STI diagnosis in the past year, using logistic regression.

Results Of 457 MSM included (20\% non-white, mean[IQR] age 33[13]), 130 (29\%), 167 (37\%) and 184 (40\%) reported $\geq 2$ CLS partners, discordant CLS and diagnosed STI respectively. Fifty-nine men (13\%) had depressive symptoms; 78\% of whom were not receiving treatment for depression. Adjusting for age, non-white ethnicity, university education, having a stable partner and recruitment region, depressive symptoms were associated with $\geq 2$ CLS partners [adjusted OR (95\% CI): 1.83 (1.01, 3.31), $\mathrm{p}=0.048]$, discordant CLS $[2.67(1.49,4.77), \mathrm{p}=0.001]$ and diagnosed STI [2.03 $(1.13,3.63), \mathrm{p}=0.017]$.

Discussion/conclusion Depressive symptoms are associated with CLS and recent STI among MSM. Management of mental health may play a role in HIV/STI prevention, although causality cannot be inferred and other factors may influence both sexual behaviour and depression.

\section{THE SEXUAL HEALTH AND WELL-BEING OF MEN WHO HAVE SEX WITH MEN (MSM): EVIDENCE FROM BRITAIN'S NATIONAL SURVEYS OF SEXUAL ATTITUDES AND LIFESTYLES (NATSAL)}

${ }^{1}$ Catherine Mercer*, ${ }^{1}$ Philip Prah, ${ }^{1}$ Clare Tanton, ${ }^{1}$ Nigel Field, ${ }^{1}$ Pam Sonnenberg, ${ }^{1}$ Kyle Jones, ${ }^{2}$ Anthony Nardone, ${ }^{1}$ Anne Johnson. ${ }^{1}$ University College London, London, UK; ${ }^{2}$ Public Health England, London, UK

\subsection{6/sextrans-2015-052126.8}

Background MSM continue to be disproportionately burdened by STIs and HIV, but sexual well-being is increasingly recognised as being broader than the absence of disease.

Aim To compare the sociodemographic, behavioural, and health profiles of MSM (reporting $>=1$ male partner(s), past 5 years) in Britain with men reporting sex exclusively with women (MSEW) during this time, and with MSM a decade earlier, to consider changes over time.
Methods Britain's third National Survey of Sexual Attitudes and Lifestyles (Natsal-3), a probability survey, interviewed 15,162 people aged 16-74 years (6,293 men) during 2010-2012 using computer-assisted personal-interviewing with computer-assisted self-interviewing for the more sensitive questions. Natsal-2, completed a decade earlier used a similar methodology.

Results Among all men in Natsal-3, 2.6\% ( $\mathrm{n}=190)$ were MSM, of whom $52.5 \%$ identified as gay. Relative to MSEW, MSM were more likely to report recreational drug use $(38.4 \%$ vs. $15.7 \%)$, treatment for depression $(14.2 \%$ vs. $5.8 \%)$, health condition (s) they perceived affected their sexual activity/enjoyment $(26.1 \%$ vs. 15.3\%), dissatisfaction with their sex life (26.3\% vs. 16.2\%), and STI diagnosis/es (past 5 years; $16.0 \%$ vs. 3.7\%). MSM reported larger numbers of partners than MSEW in all timeframes considered, differences that remained in multivariable analyses. No changes in MSM prevalence, profile, or behaviour were observed between Natsal-2 and Natsal-3.

Conclusion Poor sexual and mental health is more common among MSM than MSEW. There is thus an urgent need for health promotion among MSM that includes, but goes beyond, focusing on STI/HIV risk reduction and which is appropriate regardless of sexual identity.

\section{BEYOND MEDICAL MANAGEMENT: THE VALUE OF PUBLIC HEALTH CONTROL MEASURES IN RESPONSE TO A HIGH RISK MSM SEXUALLY TRANSMITTED INFECTION CLUSTER}

${ }^{1}$ Heather Anderson*, ${ }^{2}$ Chris Lovitt, ${ }^{3}$ Yaccub Enum, ${ }^{1}$ Andy Williams. ${ }^{1}$ Barts Health NHS Trust, London, UK; ${ }^{2}$ London Borough of Tower Hamlets, London, UK; ${ }^{3}$ London Borough of Waltham Forest, London, UK

\subsection{6/sextrans-2015-052126.9}

Background/introduction In April 2014, detailed partner notification of a male patient with acute HIV, Chlamydia and gonorrhoea identified 27 different men linked to a single residential address, having listed it as their own contact address or by being the sexual partner of someone who had. Of the 27 , several had attended GU services in the preceding three months with features common to their presentations including: high STI rates, selling of sex, adult film work, sex parties, chemsex, use of PEP and HIV seroconversion in the previous 12 months.

Methods The outbreak control team included a health adviser, GUM consultant, PHE health protection specialist and local authority public health. An implementation strategy was developed with immediate control measures and longer term service planning and development. A literature search established an STI outbreak linked to a single household to be a new precedent.

Results Immediate control measures:

- Outreach visit to and confidential inquiries of the residence

- Targeted messages on MSM apps

- Assuring consistent use of Treatment as Prevention

- MDT education on current MSM trends with enhanced training for health advisers

- Addition of a drugs worker clinic

Service development:

- GU clinic needle exchange

- Improved electronic patient record data output

- Comprehensive analysis of local MSM population

- Improved engagement with commissioning and drug/alcohol services 
Discussion/conclusion As the trend of chemsex and sex parties continues, it is likely there will be an increase in STIs linked to households. Better geospatial analysis of STI trends and collaborative working with public health is essential for rapid identification and control of outbreaks.

\section{MSM REPORT HIGH USE OF CLUB DRUGS WHICH IS ASSOCIATED WITH HIGH RISK SEXUAL BEHAVIOUR}

${ }^{1}$ Thomas Kurka*, ${ }^{2}$ Suneeta Soni, ${ }^{2}$ Daniel Richardson. 'Brighton and Sussex Medical School, Brighton, UK; ${ }^{2}$ Brighton and Sussex University Hospitals NHS Trust, Brighton, UK

\subsection{6/sextrans-2015-052126.10}

Background/introduction The prevalence of club drug use in men who have sex with men (MSM) locally is unknown but likely associated with poor sexual health. Locally there is a large MSM population with high rates of HIV and STIs.

Aim(s)/objectives The aims of this study were to quantify club drug use in MSM locally, examine differences by HIV status and identify any association between club drug use and sexual behaviour.

Methods Patient survey of MSM attending three MSM-services (STI clinic, NGO, primary care centre) in the City. We asked MSM to report ever and recent (past month) drug use. Data were analysed using SPSS.

Results 246 MSM completed surveys from January-March 2014. The median age was 35 years (18-79). 12.7\% were HIVpositive, $61.1 \%$ HIV-negative, $20.0 \%$ unsure and $5.7 \%$ never tested. The overall ever: recent club drug use was: 52.4\%:21.5\% cocaine, 49.4\%:17.1\% MDMA, 37.7\%:19.3\% mephedrone, 35.5\%:10.5\% ketamine, 24.2\%:11.0\% GHB/GBL, and 10.4\%:2.8\% crystal meth. HIV-positive MSM reported significantly higher crystal meth (Ever:37.0\% v 6.9\%: p < 0.05; Recent 13.6\%:1.3\%: p < 0.05) and GHB/GBL (Ever:48.1\% v 21.2\%: p < 0.05; Recent: 27.3\%:8.9\%: p < 0.05) use than HIV-negative/unknown. HIV-positive were significantly more likely to have injected (Slamming) club drugs ever than HIV-negative/unknown (Ever: 22.2\% v 2.5\%: p < 0.05). HIV-positive MSM using club drugs reported significantly higher rates of unprotected anal intercourse (in past 6-months) than HIV-negative/unknown (87.1\% v 57.1\%: p < 0.05).

Discussion/conclusion Club drugs use among MSM overall is worryingly high locally. In particular, HIV-positive MSM use more crystal meth and GHB/GBL, and these men are more likely to engage in unprotected anal intercourse. These data are sobering and serve as a reminder that STI and drug services should work together.

\section{CHEMSEX AND THE CITY: SEXUALISED SUBSTANCE USE IN GAY BISEXUAL AND OTHER MEN WHO HAVE SEX WITH MEN}

${ }^{1}$ Ming Lee*, ${ }^{1}$ Aseel Hegazi, ${ }^{1}$ Alison Barbour, ${ }^{2}$ Bavithra Nathan, ${ }^{2}$ Simon Green, ${ }^{2}$ Richard Simms, ${ }^{1}$ Mark Pakianathan. ${ }^{1}$ The Courtyard Clinic, Wandswort Integrated Sexual Health, St George's Healthcare NHS Trust, London, UK; ${ }^{2}$ Department of Sexual Health, The Wolverton Centre, Kingston Hospital NHS Foundation Trust, London, UK

\subsection{6/sextrans-2015-052126.11}

Background/introduction Sexualised substance use (chemsex) is an emergent phenomenon amongst some gay, bisexual and other men who have sex with men (GBMSM).
Aim(s)/objectives To describe patterns of chemsex and clinical characteristics of GBMSM attending two London sexual health clinics.

Methods Retrospective case-notes review. Data on demographics, chemsex practices, sexual behaviour, STI diagnoses and HIV status extracted from a new holistic standardised profoma used in GBMSM clinics June to December 2014.

Results 27\% $(\mathrm{n}=127)$ of 531 cases disclosed drug use. 59\% $(\mathrm{n}=73 / 124)$ reported chemsex, 13\% ( $\mathrm{n}=15 / 116)$ injected. Drugs: Mephedrone ( $\mathrm{n}=48)$, GHB/GBL $(\mathrm{n}=38)$, Crystal Meth $(\mathrm{n}=28)$ and Cocaine $(\mathrm{n}=8) .1 / 3$ disclosed $>$ one chemsex session/month. Chemsex was significantly associated with the risk taking behaviours transactional sex, group sex, fisting, sharing sex toys, HIV and hepatitis sero-discordancy $(\mathrm{p}<0.05)$, more reported sexual partners (median 3 vs. 2 in past 3 months; $\mathrm{P}<0.0001$ ) and HIV positivity (35\% vs $7 \% \mathrm{p}<0.0001)$. STIs were diagnosed more frequently in chemsex participants; Gonorrhoea $(39 \%$ vs. $6 \%$ p $<0.0001)$, Chlamydia $(11 \%$ vs. $4 \%$ p $=$ $0.05)$, Hepatitis C $(5 \%$ vs $0.3 \% \mathrm{p}=0.03)$ and PEPSE was more frequently prescribed $(14 \%$ vs. $2 \% \mathrm{p}=0.001) .42 \%$ of patients perceived chemsex to have had an adverse consequences on their physical/ mental health or career.

Discussion/conclusion The majority of GBMSM reporting chemsex were HIV negative and many perceived negative consequences from chemsex. It was also significantly associated with risk taking behaviours, STIs, hepatitis $\mathrm{C}$ and being HIV positive. A holistic assessment of GBMSM enables the identification of opportunities for targeted prevention, health promotion and wellbeing interventions.

\section{ASSOCIATIONS BETWEEN REPEAT ATTENDANCES, SEXUALLY TRANSMITTED INFECTIONS AND CHILD SEXUAL EXPLOITATION IN UNDER 16 YEAR OLDS ATTENDING GENITOURINARY MEDICINE CLINICS}

${ }^{1}$ Christopher Ward*, ${ }^{2}$ Gwenda Hughes, ${ }^{2}$ Holly Mitchell, ${ }^{3}$ Karen Rogstad. ${ }^{1}$ Central Manchester University Hospitals NHS Foundation Trust, Manchester, UK; ${ }^{2}$ Public Health England, London, UK; ${ }^{3}$ Sheffield Teaching Hospitals NHS Foundation Trust, Sheffield, UK

\subsection{6/sextrans-2015-052126.12}

Background Child sexual exploitation (CSE) diagnoses are difficult to make, often with no symptoms or signs. Previous reports suggested that sexually transmitted infections (STIs) are a CSE marker but currently there is no evidence for this.

Aim To investigate associations between attendance patterns and STIs with CSE to refine clinic-based CSE risk algorithms.

Methods STI diagnoses among < 16 year-olds during 2012 were extracted from clinics using the genitourinary medicine clinic activity dataset (GUMCAD). Clinics with $>18$ STI diagnoses (all STIs) were contacted for recruitment. Cases were defined as patients with a confirmed, bacterial or protozoal STI. Controls were defined as age and gender matched asymptomatic patients at the same clinic without STIs. An online data collection tool was developed to capture additional CSE risk factors on cases and controls. A protocol was created to aid CSE definition and stratification.

Results During 2012 in England, there were 12,819 attendances of young people aged 13-15 and 2337 STIs diagnosed: 1040 (44.5\%) were chlamydia, 220 (9.4\%) gonorrhoea and 67 (2.9\%) trichomonas. Of these infections $998(75.2 \%)$ were aged 15, 57 $(4.3 \%)$ were $\leq 13$ and 1188 (89.5\%) were female. 44 clinics had $>18$ STIs in $<16$ s, and 21 were recruited to the study. 